\title{
LOS TRIGONIIDAE DE LA COLECCION VICTOR BENAVIDES, DEPOSITADOS EN LA UNIVERSIDAD RICARDO PALMA
}

\begin{abstract}
RESUMEN
Los 14 Trigoniidae de la colección Victor Benavides Cáceres (Cretáceo de la región de Cajamarca), donada por el Museum of ?Buchotrigonia (Buchotrigonia) abrupta (von Buch, 1839); Buchotrigonia (Syrotrigonia) steinmanni (Lisson, 1930); Buchotrigonia (Syrotrigonia) gerthi (Lisson, 1930); Mediterraneotrigonia hondaana (Lea, 1861) var.boussingaulti Lisson, 1930; Pteritrigonia(Pteritrigonia) tocaimaana (Lea, 1840); Buchotrigonia (Syrotrigonia) Cox, 1952; Buchotrigonia Dietrich, 1938; Pteritrigonia (Pteritrigonia) Cox, 1969.
\end{abstract}

\section{SUMMARY}

14 Trigoniidae of the Victor Benavides Cáceres Collection (Cretaceous of Cajamarca), gift of New York's Museum of Natural History, are described, registred and determinated to the next taxa: ?Buchotrigonia (Buchotrigonia) abrupta (von Buch, 1839); Buchotrigonia (Syrotrigonia) steinmanni (Lisson, 1930); Buchotrigonia (Syrotrigonia) gerthi (Lisson, 1930); Mediterraneotrigonia hondaana (Lea, 1861) var. boussingaulti Lisson, 1930; Pteritrigonia (Pteritrigonia) tocaimaana (Lea, 1840); Buchotrigonia (Syrotrigonia) Cox, 1952; Buchotrigonia Dietrich, 1938; Pteritrigonia (Pteritrigonia) Cox 1969.

\section{INTRODUCCION}

Desde 1984 la Facultad de Ciencias Biológicas de la Universidad Ricardo Palma y el Consejo Nacional de Ciencia y Tecnología, a través de su Programa de Apoyo al Investigador, han estimulado las investigaciones sobre Trigoniidae peruanos (Alleman, 1985 a, 1985 b, 1986 a, 1986 b, 1986 c, 1989), durante las cuales fueron creados nuevos taxones y fue actualizada la taxonomía de los Trigoniidae peruanos.

En el curso de estas investigaciones se recibieron colecciones de ejemplares «tipos» del British Museum of Natural History de Londres (Reino Unido), del Museum d' Histoire Naturelle de Paris (Francia), del Institüt für Palantologie de la Universidad de Bonn (Alemania), del Smithsonian Institution de Washington (U.S.A.), del Servicio Nacional de Geología y Minería de Chile. Ultimamente se recibió una colección de fósiles lamelibranquios de V. Benavides, donación del American Museum of Natural History de Nueva York (U.S.A.)

Hasta la fecha la colección V. Benavides de la Universidad Ricardo Palma viene a ser la única colección de moluscos bivalvos del Cretáceo de Cajamarca, debidamente descrita, registrada, determinada y accesible a los investigadores. Las mencionadas especies de Trigonias han sido originalmente descritas en los trabajos de von Buch (1839), Lea (1840) y Lisson (1930) y fueron mencionadas en numerosos trabajos de correlaciones geológicas peruanas.
Se agredece al Dr. Norman Newell del Museo de Historia Natural de Nueva York, al Dr. Victor Benavides por la donación de la colección, al CONCYTEC por el apoyo brindado a nuestras investigaciones y por la donación de los muebles necesarios a la exposición permanente de los fósiles determinados en el sección Paleobiología del Museo de Historia Natural de la Universidad Ricardo Palma.

\section{PARTE TAXONOMICA}

I. Clase Bivalvia Linné, 1758 (Buonanni, 1681) (según Cox, 1969)

Subclase Palaeoheterodonta Newell, 1965

Orden Trigonoidea Dall, 1889.

Superfamilia Trigoniacea Lamarck, 1819

Familia Trigoniidae Lamarck, 1819

Género Buchotrigonia Dietrich, 1938

VBRP 22, VBRP 23, VBRP 24 (VB-112) ?Buchotrigonia (Buchotrigonia) abrupta (von Buch, 1839).

Tres ejemplares, de los cuales el mejor conservado se presenta como un individuo completo de valvas cerradas, la valva derecha está dañada en la mayor parte de su superficie, la valva izquierda tiene las costas bastante gastadas, de tal manera que no se pueden apreciar la existencia de granulación por medio de las estrias de crecimiento. Tampoco se nota con suficiente nitidez los 


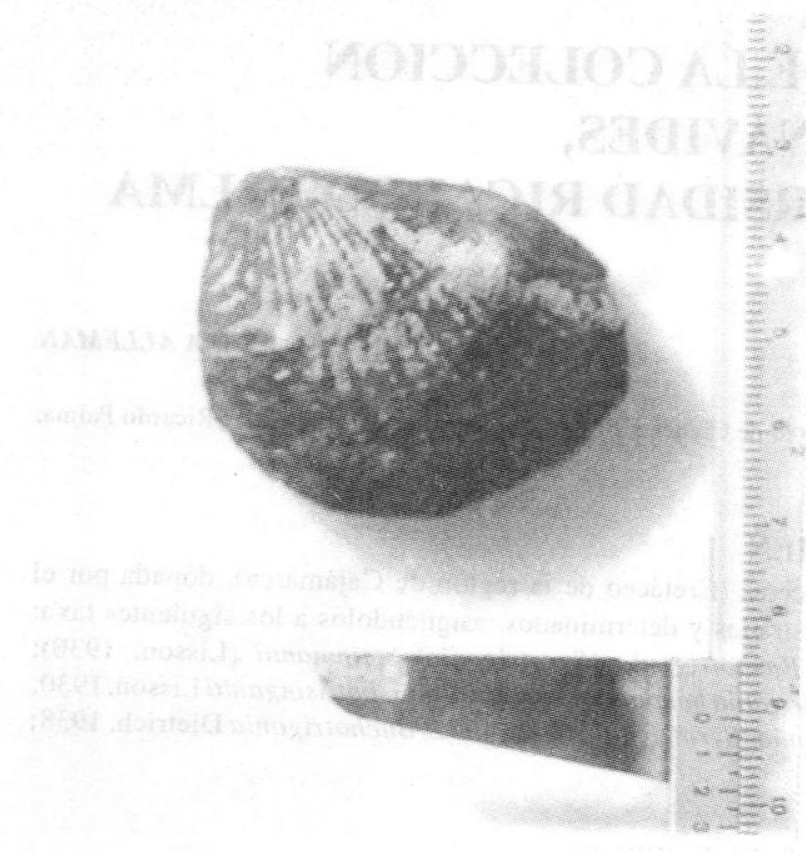

Fig. 1. ? Buchotrigonia (Buchotrigonia) abrupta (von Buch, 1838). Vista de la valva izquierda. VBRP22.

detalles de la ornamentación de las partes distantes del área y de los flancos. Sin embargo, colocamos los ejemplares provisoriamente en la mencionada especie por su forma triangular, más larga que alta, inequilateral, con umbos opistógiros; área ancha, cuya superficie forma en la región umbonal un ángulo casi recto; la ornamentación del área consta de costas oblícuas; carena marginal mal definida, roma, excepto en el tercio umbonal y en el flanco ornamentado con tres grupos de costas: excéntricas, verticales y concéntricas. La duda se presenta debido a que, por el desgaste de la superficie de la concha, persisten como huellas las costas oblícuas delárea que parecen extenderse más allá del tercio anterior y las costas verticales no parecen volverse obsoletas en la extremidad posterior de la valva, como es el caso del género Buchotrigonia y de la especie abrupta según von Buch (1839) y Dietrich (1938).

Dimensiones VBRP 22: largo: $46.8 \mathrm{~mm}$. alto : $39.4 \mathrm{~mm}$.

espesor aproximativo de las dos valvas: $28.9 \mathrm{~mm}$.

VBRP 25: Buchotrigonia (Syrotrigonia) steinmanni (Lisson, 1930). Ejemplar relativamente pequeño. Se presenta abierto, ambas valvas unidas a nivel de la charnela, escudete y área.

VBRP 26: Buchotrigonia (Syrotrigonia) steinmanni (Lisson, 1930). Valva izquierda casi completa con dientes parcialmente visibles.

VBRP 27: Buchotrigonia (Syrotrigonia) gerthi (Lisson, 1930).

Ejemplar relativamente pequeño y muy alterado.
VBRP 28: Buchotrigonia (Syrotrigonia) gerthi (Lisson, 1930).

Parte umbonal de ambas valvas unidas.

VBRP 29: Buchotrigonia (Syrotrigonia) sp. Cox, 1952.

Valva izquierda incompleta, presentando parcialmente la dentądura. 1952.

VBRP 30: Buchotrigonia (Syrotrigonia) sp. Cox, Fragmento umbonal.

VBRP 31: Buchotrigonia sp. Dietrich, 1938. Molde interno.

Género Mediterraneotrigonia Nakano, 1974.

VBRP 32 (VB-125) Mediterraneotrigonia hondaana (Lea, 1861) var. Boussingaulti Lisson, 1930. Ejemplar: casi completo.

Dimensiones: largo: $72.5 \mathrm{~mm}$. alto : $66.3 \mathrm{~mm}$.

espesor aproximativa de las dos valvas: $39.5 \mathrm{~mm}$.

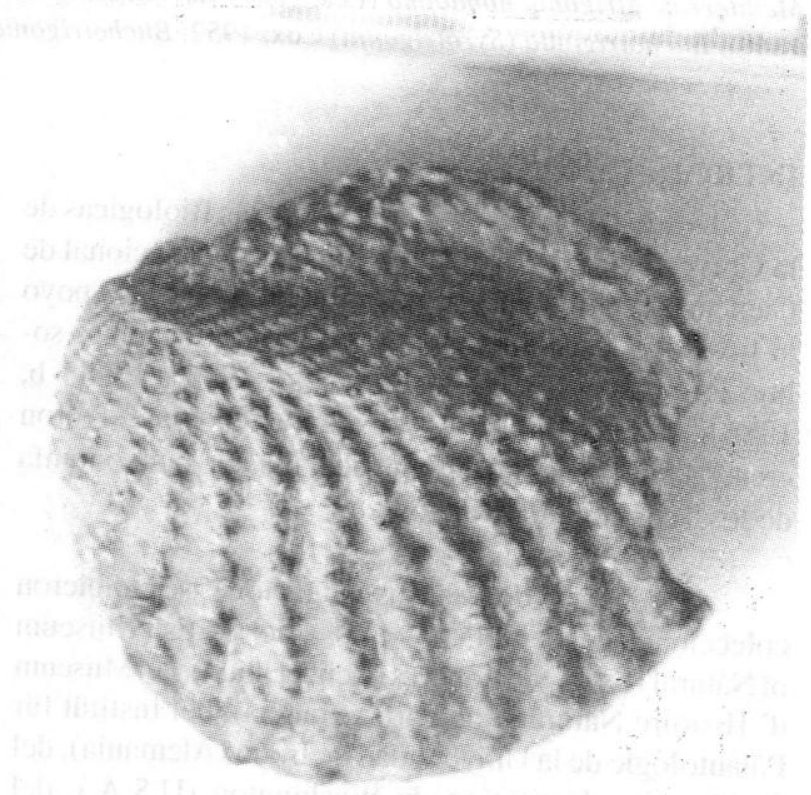

Fig. 2. Medilerraneotrigonia hondaana (Lea, 1861) var. boussingaulti Lisson, 1930 . Vista de la valva izquierda. VBRP32

Género Pteritrigonia van Hoepen, 1929.

VBRP 34, VBRP 35 (VB-112) Pteritrigonia (Pteritrigonia) tocaimaana, (Lea, 1840).

Dos hermosos ejemplares.

$\begin{array}{ccc}\text { Dimensiones: } & \text { VBRP 34, } & \text { VBRP 35 } \\ \text { Largo: } & 70.9 \mathrm{~mm} . & 68.0 \mathrm{~mm} . \\ \text { Alto: } & 60.9 \mathrm{~mm} . & 60.9 \mathrm{~mm} .\end{array}$

Espesor aproximada de 2 valvas:
$45.4 \mathrm{~mm}$. 


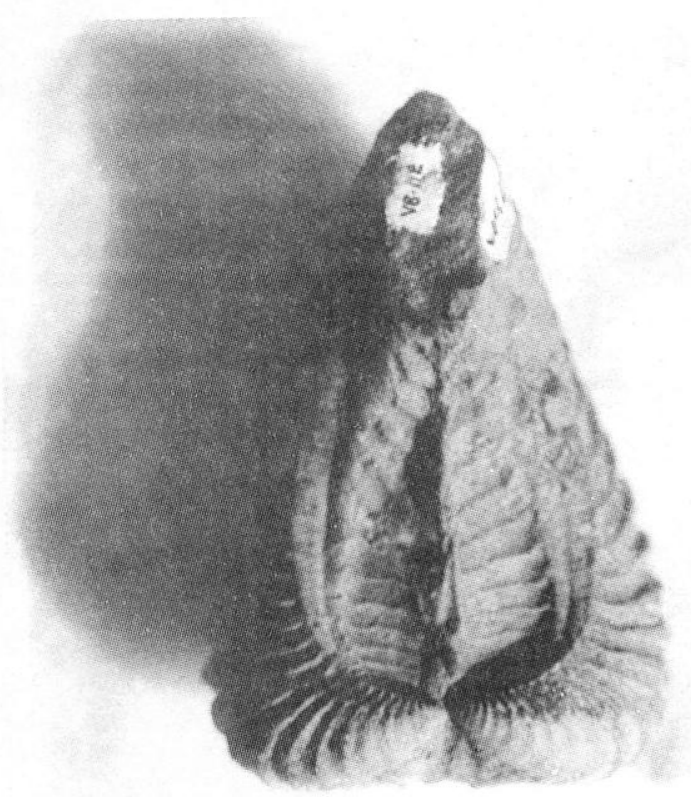

Fig. 3. Pteritrigonia (P'teritrigonia) tocaimaana (Lea, 1840) vista del área VBRP34.

\section{REFERENCIAS BIBLIOGRAFICAS}

ALLEMAN, V. 1985a. Virgotrigonia. género nuevo de Trigoniidae Lamarck, 1819 (Mollusca, Bilvavia). Bol. Soc. Geol. Perú, 75: 1-11.

ALLEMAN, V. 1985b. Investigaciones sobre Trigoniidae peruanos. Bol.Fac.Cienc. Biológicas Universidad Ricardo Palma, 1: 16-20.

ALLEMAN, V., 1986a. Una colección de Duplicata de «Tipos» de Trigoniidae en la Universidad Ricardo Palma. Bol.Fac.Cienc. Biológicas Universidad Ricardo Palma, 3: 23-29.

ALLEMAN, V., 1986b. Una Recolección de Moluscos fósiles en el Distrito de Huaylas, Departamento de Ancash. Bol. Fac. Cienc. Biológicas. Universidad Ricardo Palma, 3: 3037.

ALLEMAN, $V$., 1986c. Trigoniidae del Perú: Datos bibliográficos de las Localidades, de la Fauna asociada, de la Estratigrafía y de la Presencia de Tipos en las Colecciones de Lima. Revista Universidad Ricardo Palma, 8/9: 111-134.

ALLEMAN, V., 1989. LaColección de Moluscos Lamelibranquios del Cretáceo del Norte del Perú depositada en la Facultad de Ciencias Biológicas de la Universidad Ricardo Palma (Lima). Workshop sobre el Cretáceo del Perú. Cretáceo de América Latina IUGS-UNESCO, 242: 21-23.

BENAVIDES, V. 1956. Cretaceous System of Northern Peru. Bull. Amer. Mus. Nat. Hist., 108: 353-394.

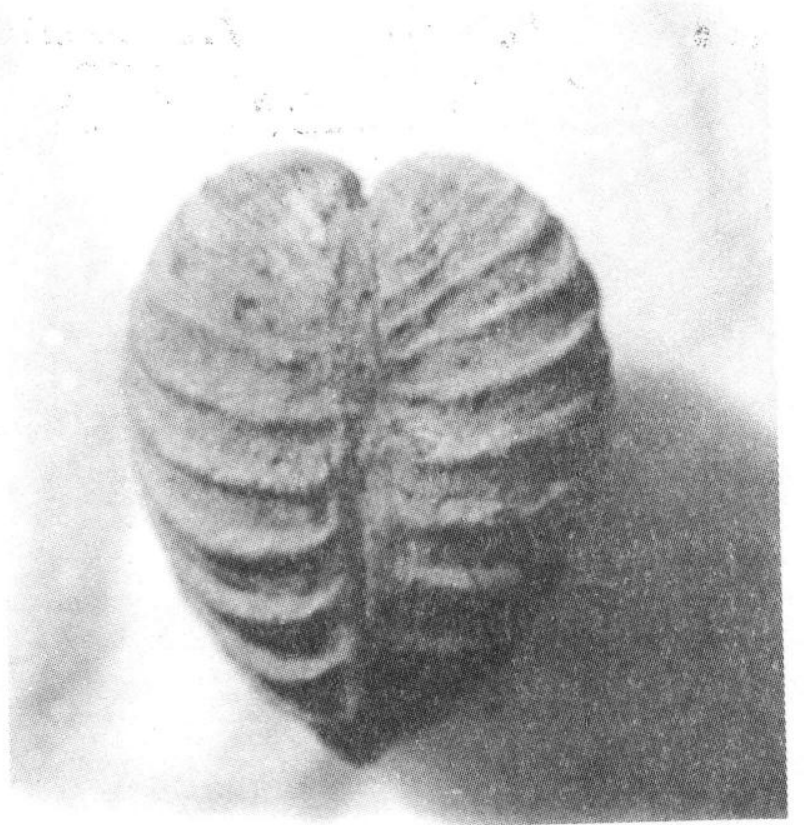

1.13. 4. P'teritrigonia (P'teritrigonia) tocaimaana (Lea, 1840) a. Vista frontal. VBRP.3.

b. Vista de la valva derecha VBRP3.5.

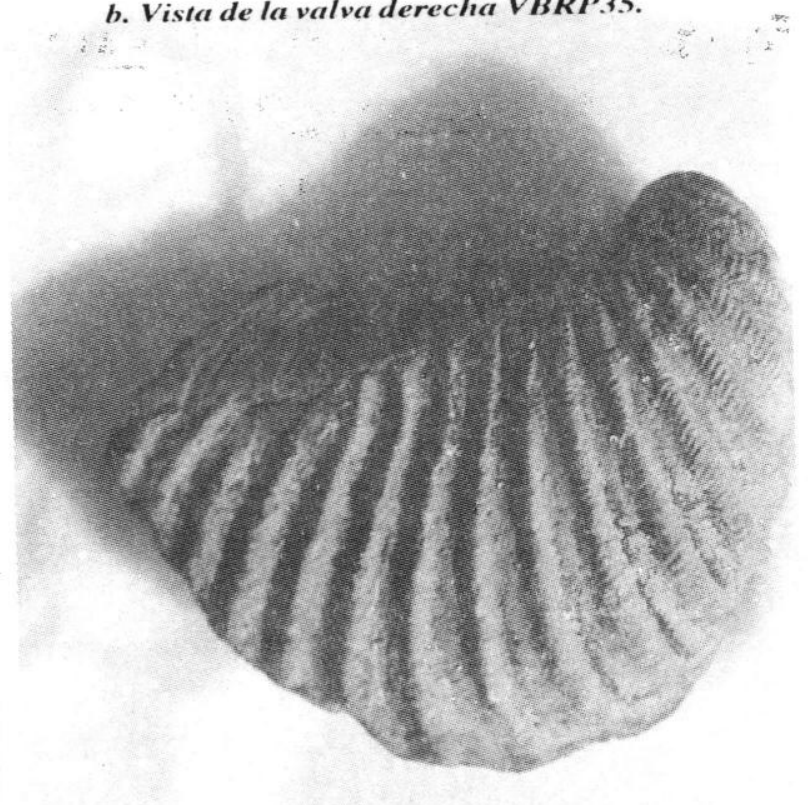

$\boldsymbol{B} \boldsymbol{U} \boldsymbol{C H}, \boldsymbol{L}$. von, 1839. Petrifications recueillies en Amerique.

COX, L. 1952, Notes on the Trigoniidae, with outlines of a classification of the Family. Malacological Soc. London, 29: 45-70.

COX, L. 1969, Family Trigoniidae Lamarck, 1819, en Treatise of Invertebrate Paleontology. Part. $\mathrm{N}^{\circ} 1$, Mollusca 6. Bivalvia. Geol. Soc. America y Kansas Univ.

DIETRICH, W., 1938. Lamelibranquios cretácicos de la Cordillera Oriental. Estudios Geol. Pal. Cordillera Oriental, Colombia, Parte 3: 81-108.

LISSON, C. 1930, Contribución al Conocimiento de algunas Trigonias neocomicas del Perú. Bol. Minas Ind. Constr. Lima, Serie 2., 20: 3-26. 